\title{
Evaluation of Microbolometer-Based Thermography for Gossamer Space Structures
}

\author{
Jonathan J. Miles ${ }^{*}$, Joseph R. Blandino ${ }^{\mathrm{a}}$, Christopher H. M. Jenkins ${ }^{\mathrm{b}}$, Richard S. Pappa ${ }^{\mathrm{c}}$, \\ Jeremy Banik ${ }^{\mathrm{b}}$, Hunter Brown ${ }^{\mathrm{a}}$, Kiley McEvoy ${ }^{\mathrm{a}}$ \\ ${ }^{\mathrm{a} J a m e s}$ Madison University, Harrisonburg, VA 22807 \\ ${ }^{\mathrm{b}}$ South Dakota School of Mines and Technology, Rapid City, South Dakota 57701 \\ 'NASA Langley Research Center, Hampton, Virginia 23681
}

\begin{abstract}
In August 2003, NASA's In-Space Propulsion Program contracted with our team to develop a prototype on-board Optical Diagnostics System (ODS) for solar sail flight tests. The ODS is intended to monitor sail deployment as well as structural and thermal behavior, and to validate computational models for use in designing future solar sail missions. This paper focuses on the thermography aspects of the ODS. A thermal model was developed to predict local sail temperature variations as a function of sail tilt to the sun, billow depth, and spectral optical properties of front and back sail surfaces. Temperature variations as small as $0.5^{\circ} \mathrm{C}$ can induce significant thermal strains that compare in magnitude to mechanical strains. These thermally induced strains may result in changes in shape and dynamics. The model also gave insight into the range and sensitivity required for in-flight thermal measurements and supported the development of an ABAQUS-coupled thermo-structural model. The paper also discusses three kinds of tests conducted to 1) determine the optical properties of candidate materials; 2) evaluate uncooled microbolometer-type infrared imagers; and 3 ) operate a prototype imager with the ODS baseline configuration. (Uncooled bolometers are less sensitive than cooled ones, but may be necessary because of restrictive ODS mass and power limits.) The team measured the spectral properties of several coated polymer samples at various angles of incidence. Two commercially available uncooled microbolometer imagers were compared, and it was found that reliable temperature measurements are feasible for both coated and uncoated sides of typical sail membrane materials.
\end{abstract}

Keywords: optical, diagnostics, gossamer, microbolometer, uncooled, infrared, membrane, temperature, solar sails

\section{INTRODUCTION}

An important aspect of evaluating the in-space performance of membrane structures, particularly during early test flights, will be monitoring the temperature of the membrane itself. Membrane temperature monitoring is necessary to validate thermal models, evaluate the performance of membrane coatings, and understand and evaluate deviations in the predicted shape and dynamic performance of the sailcraft. Temperature variations across the membrane may result from non-uniform heating caused by the orientation of the sailcraft with respect to the sun and variations in the optical properties of the membrane coatings. Resultant temperature variations across the membrane would cause non-uniform expansion or contraction of the membrane because the coefficient of thermal expansion of the membrane material is non-zero. This may cause, for example, slack regions or an asymmetric membrane shape. Both the attitude control characteristics and thrust of a solar sail are affected by changes in membrane shape. Therefore, knowledge of the membrane temperature profile is necessary in order to predict membrane shape and associated performance characteristics. Characterization of the membrane temperature profile is a necessary first step in designing an effective measurement system. In order to gain insight into the nominal temperature profile, a thermal model was developed. This paper presents the development of a thermal model for predicting membrane temperatures, an overview of thermal measurement technologies, and a presentation and discussion of test results to date.

* milesjj@jmu.edu; phone +1 540568 3044; fax +1 540568 2761; http://www.isat.jmu.edu/miles.htm; College of Integrated Science and Technology, MSC 4102, James Madison University, Harrisonburg, VA 22807 


\section{THERMAL MODELING OF SOLAR SAILS}

A thermal model is a necessary tool for designing a thermal measurement system. The model provides insight into the range and sensitivity needed for the measurement instrument. When developing a thermal model for a sailcraft, several sources of thermal energy may be considered depending on the sailcraft design and mission profile. Heating may come from on-board power systems, the sun, from a nearby planet, or solar energy reflected from a nearby planet (albedo radiation). ${ }^{1,2,3,4}$ This section describes the development of a thermal model that can be used to support the design of a thermal measurement system for a solar sail flight validation mission.

Radiation is the dominant heat transfer mode for membrane structures in space. Conduction is minor because the thermal conductivity of typical membrane materials (Kapton, CP-1, and Mylar) is relatively small and the conduction path is infinite, and convection is negligible. In order to design an effective measurement system, it is important to understand the nature of the temperature variations across the membrane and how they are impacted by the influx of energy from the sun. Only a small portion of the electromagnetic spectrum influences the thermal energy of an object, and matter emits thermal radiation between 0.1 and $100 \mu \mathrm{m}$. The thermal radiation spectrum is comprised of part of the ultraviolet spectrum $(0.01$ to $0.4 \mu \mathrm{m})$, the visible spectrum $(0.4$ to $0.8 \mu \mathrm{m})$ and the infrared spectrum. Infrared radiation is emitted at wavelengths between 0.8 and $100 \mu \mathrm{m}$. The balance of electromagnetic energy emitted and absorbed in the infrared portion of the spectrum has the greatest effect on the temperature of an object.

A blackbody emits and absorbs radiation uniformly in all directions. The energy emitted by a real object per unit time, per unit area, at a specific wavelength, $\lambda$, and in a particular direction, $\theta$, is a fraction of the blackbody radiation, $E_{b}$, as described by Equation 1, where $0 \leq \varepsilon \leq 1$ and $\sigma$ is the Stefan-Boltzmann constant. The dependence on wavelength and direction are more significant than the temperature dependence, and any variation in emittance would be modest assuming an operating temperature, $T$, on the order of $50^{\circ} \mathrm{C}$.

$$
\dot{q}(\lambda, \theta, T)=\varepsilon(\lambda, \theta, T) E_{b}(T)=\varepsilon(\lambda, \theta, T) \sigma T^{4}
$$

The sun can be treated as a disk of radius $6.95 \times 10^{5} \mathrm{~km}$ emitting as a blackbody at approximately $5780 \mathrm{~K}^{5}$ The solar radiation at any point in space can be calculated from the inverse square law, thus suggesting that at $1.5 \times 10^{8} \mathrm{~km}$ $(1 \mathrm{AU})$ the solar radiation per unit area is approximately $1370 \mathrm{~W} / \mathrm{m}^{2}$. Although the sun behaves similar to a blackbody and thus radiates energy uniformly in all directions, this is not true for man-made objects such as membranes. The optical properties of a membrane material or surface coating will determine if an average value of emittance is appropriate for thermal analysis, or whether an effective value must be determined by integrating across the spectrum of emission and absorption.

The temperature of a membrane is a function of the rate at which thermal radiation is absorbed and emitted by the material. Incident thermal radiation may be absorbed by the material, reflected by the membrane surface coating, or transmitted through the membrane. The summation of the three corresponding optical property values must equal unity; that is energy must be conserved as described by Equation 2 in which $\alpha, \rho$, and $\tau$ represent absorptance, reflectance, and transmittance, respectively. Note that the optical properties are a function of wavelength and incident angle. Values for reflectance and transmittance were measured in the laboratory as described in the next section. The absorptance is then obtained by substitution into Equation 2, and Kirchhoff's Law is applied to determine emittance as shown by Equation 3. ${ }^{6,7}$

$$
\begin{aligned}
& \alpha(\lambda, \theta)+\rho(\lambda, \theta)+\tau(\lambda, \theta)=1 \\
& \varepsilon(\lambda, \theta)=\alpha(\lambda, \theta)
\end{aligned}
$$

When thermal radiation is exchanged between a surface and much larger isothermal surroundings, such as between a membrane and the space environment, the energy balance is governed by the solar flux and the radiation input from the surroundings and that emitted by the membrane into the surroundings. Equation 4 describes this energy balance in which $G$ represents the incident radiation originating from the surroundings. 


$$
\dot{q}_{\text {sun }}+G \alpha(\lambda, \theta)=\varepsilon(\lambda, \theta) \sigma T^{4}
$$

By substitution of Equation 3 into Equation 4 and rearranging terms, assuming that the background (deep space) is approximated as a blackbody at temperature $T_{\infty}$, Equation 5 is derived and describes the energy balance for a given wavelength and direction.

$$
\dot{q}_{\text {sun }}=\varepsilon(\lambda, \theta) \sigma T^{4}-\varepsilon(\lambda, \theta) \sigma T_{\infty}^{4}=\varepsilon(\lambda, \theta) \sigma\left(T^{4}-T_{\infty}^{4}\right)
$$

The steady state temperature of a solar sailcraft oriented perpendicular to the solar vector has been presented previously by Equation 6 in which the subscripts 1 and 2 refer to the sunward and shadow sides of the sail membrane, respectively, and the emittance and absorptance values are averaged across all wavelengths. ${ }^{8.9}$

$$
T^{4}=\frac{\alpha_{1} \dot{q}_{\text {sun }}}{\left(\varepsilon_{1}+\varepsilon_{2}\right) \sigma}
$$

A typical four-quadrant sail is shown in Figure 1. A set of equations are derived below that are used to predict the temperature distribution across a sail membrane that is billowed due to solar pressure and oriented at a pitch angle with respect to the solar vector. The model considers one quadrant of a four-quadrant sail. The following assumptions are made: (1) the thermal conductivity of the membrane material is negligible; (2) the sail surface is not necessarily flat; (3) radiation exchange between elements on the sail surface is negligible; (4) there is no temperature gradient through the thickness of the membrane; and (5) the background space environment is isothermal at $3 \mathrm{~K}$.

The first assumption was considered previously. The second is based on the fact that the sail tension will be low and solar pressure will cause the sail to billow. The third assumption arises because the sun-facing side of the sail membrane will have a highly reflective coating, typically $\rho>0.9$, and therefore absorption of radiation will be very low. In addition, the view factors between membrane elements are infinitesimally small. Sail membranes are thin, typically less than $5 \mu \mathrm{m}$, thus thermal gradients do not develop across the thickness. Although the $3 \mathrm{~K}$ background temperature of space is typically neglected, it is advantageous to retain this term in order for the model to be practical for predicting temperatures during ground tests in which the background temperature will be much greater than $3 \mathrm{~K}$.

As an example, a 70-m square sail with a $0.7-\mathrm{m}$ billow is considered. The corners of the quadrant are pinned and the maximum billow displacement is near the center of the hypotenuse of the quadrant. The membrane is divided into a mesh as shown in Figure 2, superimposed on a contour of membrane shape. The coarseness of the mesh will vary depending on the shape of the deformed membrane. An energy balance is performed on each element independently. A schematic of a single element with solar orientation angles is shown in Figure 3. The energy balance for a single element is given by Equation 7 .

$$
\alpha_{1} \dot{q}_{\text {sun }} A_{\text {element }} \cos \psi \cos \phi-\varepsilon_{1} A_{\text {element }} \sigma\left(T^{4}-T_{\infty}^{4}\right)-\varepsilon_{2} A_{\text {element }} \sigma\left(T^{4}-T_{\infty}^{4}\right)=\gamma c A_{\text {element }} z_{\text {element }} \frac{d T}{d t}
$$

in which $\psi$ and $\varphi$ are the angles of incidence with respect to each planar axis, $z$ is the thickness of each element, and $\gamma$ and $c$ represent density and specific heat, respectively. Equation 7 simplifies to

$$
\alpha_{1} \dot{q}_{\text {sun }} \cos \psi \cos \phi-\left(\varepsilon_{1}+\varepsilon_{2}\right)\left(T_{\infty}^{4}-T^{4}\right)=\gamma c z_{\text {element }} \frac{d T}{d t}
$$

in which the term $\alpha_{1}$ represents the solar (short-wave) absorptance on the sunward side. In the case of a solar sail, changes in the sun-sail orientation will occur slowly. Because the density and thickness are small, the thermal response time is very short, and steady-state temperature is achieved quickly. Therefore, the right-hand side of Equation 8 is assumed zero and further reduction to Equation 9 is achieved from which sail temperature associated with any particular element, $T$, can be calculated. 


$$
\frac{\alpha_{1} \dot{q}_{s u n} \cos \psi \cos \phi}{\sigma\left(\varepsilon_{1}+\varepsilon_{2}\right)}=\left(T^{4}-T_{\infty}^{4}\right)
$$

The difference between this expression and Equation 6 is that Equation 9 accounts for a non-normal solar vector and a non-zero background temperature field. Energy is absorbed within the solar waveband ( 0.2 to $2 \mu \mathrm{m})$ but is emitted predominantly at wavelengths between 2 and $25 \mu \mathrm{m}$. Equation 9 allows for variation in sail shape and sail-sun orientation to be considered during thermal analysis.

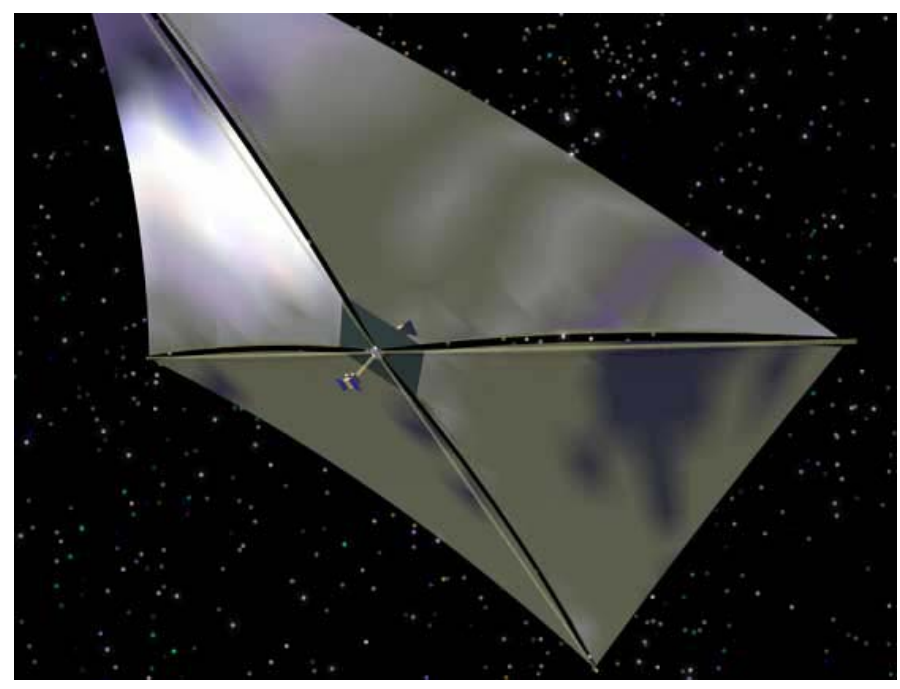

Figure 1. Four quadrant sail. (Photo courtesy of NASA.)

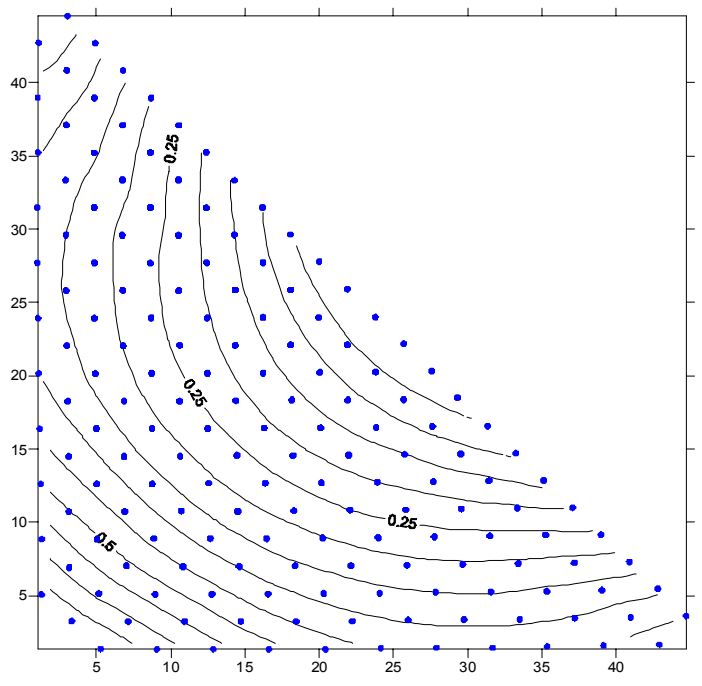

Figure 2. Mesh used for thermal analysis overlayed onto contour plot of the quadrant shape.

The application of Equations 7-9 assumes that optical properties are constant or can be approximated as constant. However, measurements have shown that some membrane coatings have considerable spectral variation in property values. Variations in coating thickness can also cause variability in optical property values. For these cases, the assumption of graybody radiant emission provides an imperfect approximation of the rate at which energy is emitted by a solar sail. This was resolved by incorporating terms into the energy balance for each side of the sail that numerically integrate the Planck distribution law with the measured spectral emittance profile, incorporated to provide a more precise estimate of energy emitted by the membrane. By application of the trapezoidal rule, the total energy emitted is estimated using Equation 10 in which $n$ represents the total number of regions and $\lambda_{i+1}-\lambda_{i}$ is the width of each waveband. Figure 4 shows the predicted temperature distribution on the sample 70-m quadrant given specific operating conditions.

$$
E_{i n}=\sum_{i=1}^{n-1}\left(\frac{\varepsilon_{i}+\varepsilon_{i+1}}{2}\right)\left(\frac{c_{1}}{\left(\frac{\lambda_{i}+\lambda_{i+1}}{2}\right)^{5}\left[\exp \left(\frac{c_{2}}{\left(\frac{\lambda_{i}+\lambda_{i+1}}{2}\right) T_{e}}\right)-1\right]}\right)\left(\lambda_{i+1}-\lambda_{i}\right)
$$




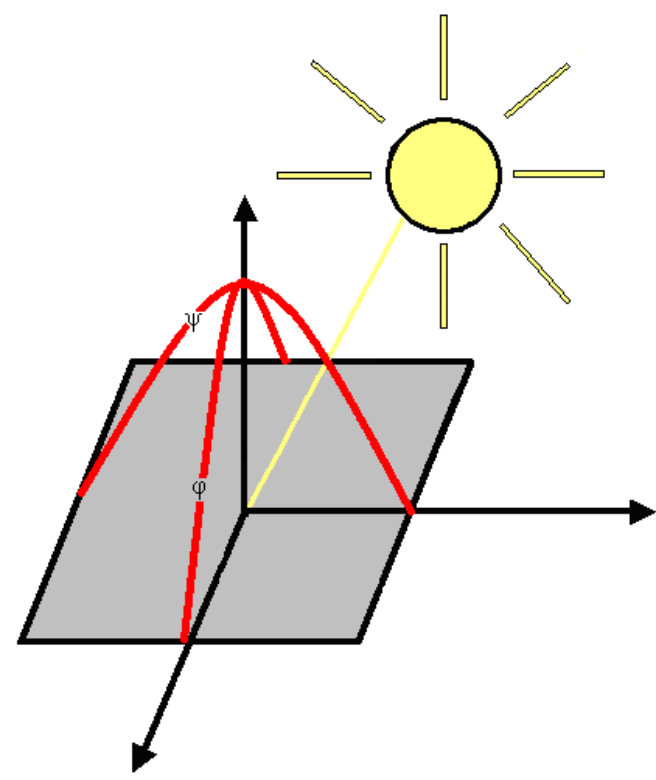

Figure 3. A schematic of a single sail element with solar orientation angles depicted.

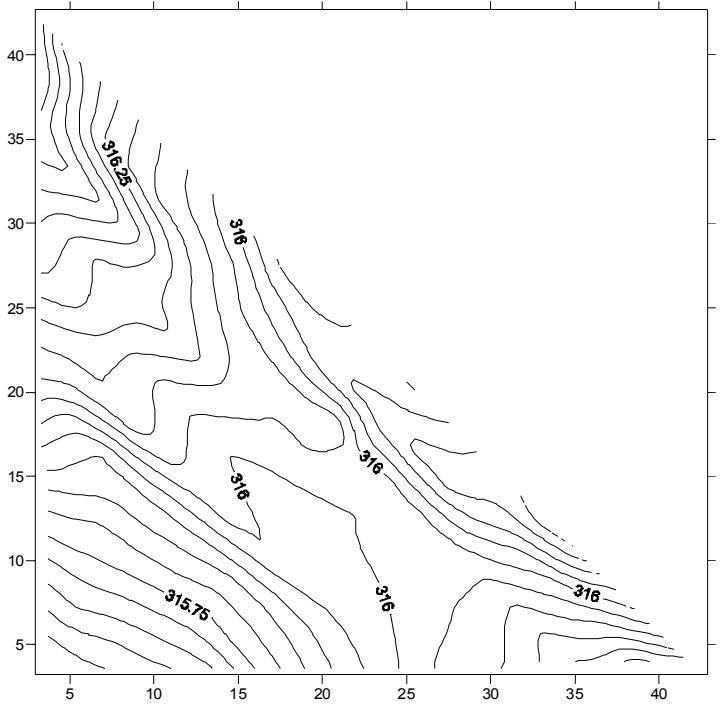

Figure 4. Temperature distribution predicted by thermal model.

\section{INFRARED MEASUREMENT TECHNIQUES}

Application of the thermal model has shown that small changes in optical property values can cause significant variations in predicted membrane temperatures and temperature differences. Solar sails are thin-film membrane structures that are very susceptible to wrinkling, which may affect sail performance or cause local regions of overheating. In addition, knowledge of the temperature field and its impact on thermal strain distribution is important for predicting and understanding in-space membrane stresses and dynamics. Significant temperature gradients across the sail surface can develop depending on variations in thickness of metal coatings, sail orientation to the sun, and the degree of billow in the sail. Such gradients can cause thermal strains on the membrane that exceed mechanical strains, which can significantly affect stresses and structural dynamics of the sail. Even minute temperature gradients, less than $0.5^{\circ} \mathrm{C}$, can cause thermal strains to exceed mechanical strains.

Polymeric materials are known to exhibit short-term (3-5 year) space environmental durability. ${ }^{10}$ However, sailcraft intended for future missions are intended to operate for greater than 10, perhaps even as long as 30 years. Thus, material property changes that occur during operation may result in unpredicted sailcraft performance. Because of their inherent toughness and flexibility, low density, thermal stability, radiation resistance, and mechanical strength, aromatic polyimides (such as CP-1 colorless polyimide) have excellent potential for use as advanced materials on large space structures. However, optical property data for such substrates, coated or not, are needed to make reliable predictions of radiative energy gains and losses from such materials. In many instances, these properties play an important role in energy conservation and control. The optical properties govern the energy interactions between the materials and their surroundings.

\subsection{CHARACTERIZATION OF OPTICAL PROPERTIES}

The surface of any material presents a unique set of optical properties. A perfect blackbody absorbs all radiation incident upon it at all wavelengths. A real surface however, absorbs only a portion of the incident radiation, and the fraction absorbed varies with wavelength as well as with temperature of the emitting surface. In addition, only a fraction of the radiative energy incident on a real surface is reflected by the surface. If a body is opaque to radiation, the sum of the reflectance and the absorptance of the body is unity in order to conserve energy according to Equation 2. The 
thermal model described previously performs an energy balance and predicts the steady-state temperature profile of a solar sail in space. The model assumes that the sunward side of the membrane absorbs solar radiation at a rate defined by an average solar absorptance value, and that the optical properties at infrared wavelengths on both sides of the membrane vary are spectrally dependent.

A Surface Optics Corporation SOC-100 Hemispherical Directional Reflectometer (HDR) coupled with a Nicolet Thermo Electron Magna-650 Fourier Transform Infrared Spectrometer (FTIR) was used to characterize the optical properties of sample membrane materials in the infrared waveband. The HDR/FTIR system is capable of measuring collimated and scattered reflectance of material samples with different polarization conditions, within a range of incident angles between $10^{\circ}$ and $80^{\circ}$, and across a spectral range between 2 and $25 \mu \mathrm{m}$. The SOC-100 measures hemispherical directional reflectance, defined as the ratio of the power reflected by a sample into a narrow solid angle to total incident power when the sample is hemispherically and uniformly illuminated. The HDR/FTIR measurement can be partitioned into two components of reflectance, specular and diffuse. A geometrical depiction of the system is provided in Figure 5.

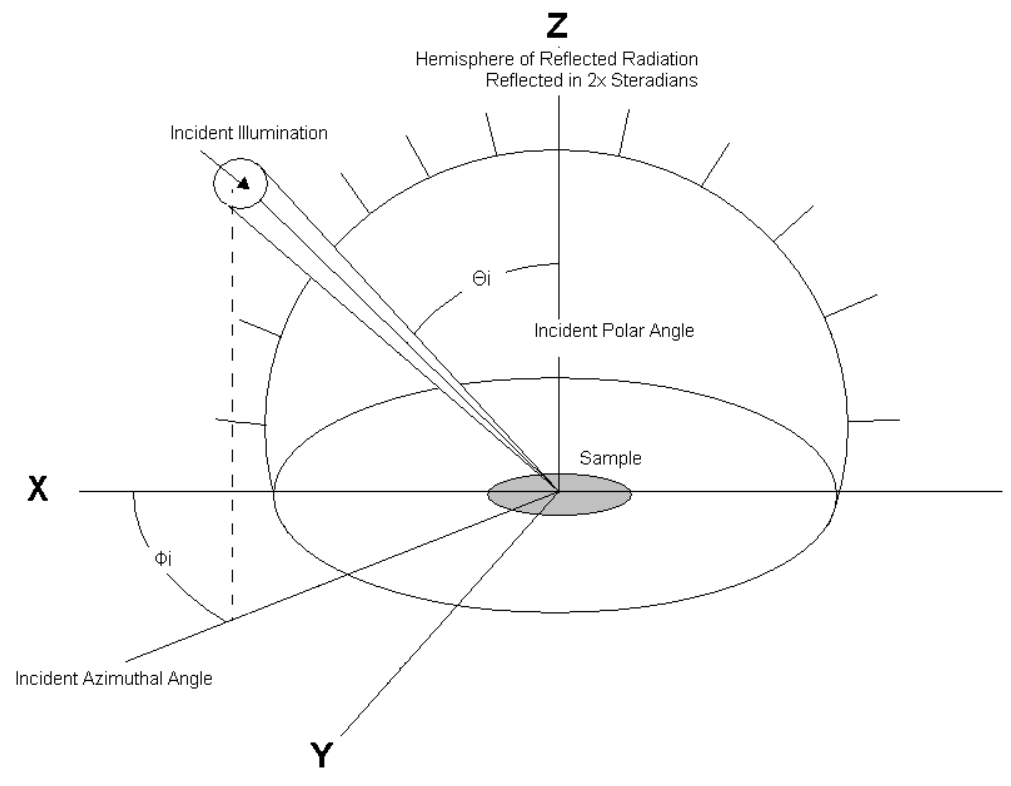

Figure 5. Geometrical description of the hemispherical directional radiometer. (Courtesy of Surface Optics Corporation.)

The specular region is the solid angle equal to the cone of illumination, while the diffuse component is the solid angle that comprises the remaining region within the hemisphere. By blocking the specular portion of the beam with a beam blocker while measuring the reflected power, the diffuse reflectance can be determined. Since energy must be conserved, the specular reflectance is calculated by subtracting diffuse reflectance from total reflectance. The SOC-100 also measures hemispherical directional transmittance, which represents the ratio of the total power transmitted through a target to the total power incident upon it. The hemispherical directional transmittance is a function of the angle of incidence, polarization, wavelength, and the material properties.

\subsection{MEMBRANE MATERIALS}

In order to best estimate membrane temperature in situ, the thermal model predicted sail temperature distribution and accounted for variation in optical properties with respect to wavelength and non-uniformities in membrane thickness, and angle between normal to the sail and the sun. Reflectance and transmittance are two important properties that are considered in the thermal analysis of sail membranes, both of which can vary with material thickness and coating and thus impact absorption and emission of infrared radiation and therefore the overall energy balance. Given the direct reflectance and transmittance measurements acquired using the HDR/FTIR, other properties of the samples were determined including directional angular and total hemispherical emittance. 
A study was conducted to characterize the optical properties of four different membrane materials. Table 1 presents the samples and their mechanical properties. Two different varieties of Mylar material were tested. The last row of Table 1 describes the type of measurement that was conducted for which HDR represents hemispherical directional reflectance and HDT hemispherical directional transmittance. ${ }^{\text {a }}$ All materials were subjected to reflectance and transmittance testing except the CP-1 material which, due to a limited availability of material, was tested only for reflectance. All samples tested for HDT were determined to be essentially opaque in the infrared; thus Equations 2 and 3 could be applied to determine spectral absorptance and emittance for each sample.

Table 1. Membrane substrates, coefficient of thermal expansion, and resulting thermal strain due to temperature differences.

\begin{tabular}{|l|llll|}
\hline Material & Kapton & $\begin{array}{l}\text { CP-1 colorless } \\
\text { polyimide }\end{array}$ & Mylar & Mylar \\
\hline Coefficient of thermal & & & & \\
expansion & $2.0 \times 10^{-5} /{ }^{\circ} \mathrm{C}$ & $3.7 \times 10^{-5} /{ }^{\circ} \mathrm{C}$ & $1.7 \times 10^{-5} /{ }^{\circ} \mathrm{C}$ & $1.7 \times 10^{-5} /{ }^{\circ} \mathrm{C}$ \\
$\varepsilon_{\text {Thermal }}$ with $\Delta \mathrm{T}$ of $0.2^{\circ} \mathrm{C}$ & $4 \mu \varepsilon$ & $7.4 \mu \varepsilon$ & $3.4 \mu \varepsilon$ & $3.4 \mu \varepsilon$ \\
$\varepsilon_{\text {Thermal }}$ with $\Delta \mathrm{T}$ of $0.5^{\circ} \mathrm{C}$ & $10 \mu \varepsilon$ & $18.5 \mu \varepsilon$ & $8.5 \mu \varepsilon$ & $8.5 \mu \varepsilon$ \\
$\varepsilon_{\text {Thermal }}$ with $\Delta \mathrm{T}$ of $1.5^{\circ} \mathrm{C}$ & $30 \mu \varepsilon$ & $55.5 \mu \varepsilon$ & $25.5 \mu \varepsilon$ & $25.5 \mu \varepsilon$ \\
front side coating & aluminum & & aluminum & aluminum \\
back side coating & uncoated & & chromium & black chrome \\
HDR/HDT & HDR/HDT & HDT & HDR/HDT & HDR/HDT \\
\hline
\end{tabular}

\subsection{SURFACE OPTICAL PROPERTY MEASUREMENTS}

Data are presented here for 1-mil-thick Kapton aluminized on one side and for 0.8-mil-thick Mylar aluminized on the proposed sun-facing side and coated with a high-emittance "black chrome" coating on the proposed shadow side. It is important to note that on the sun-facing side of the sail, energy will be absorbed at solar wavelengths $(0.2-2 \mu \mathrm{m})$, while thermal emissions will occur on both sides at infrared wavelengths (characterized here for 2-25 $\mu \mathrm{m}$ ). Figure 6 shows the emittance profile at solar wavelengths for the Kapton sample.

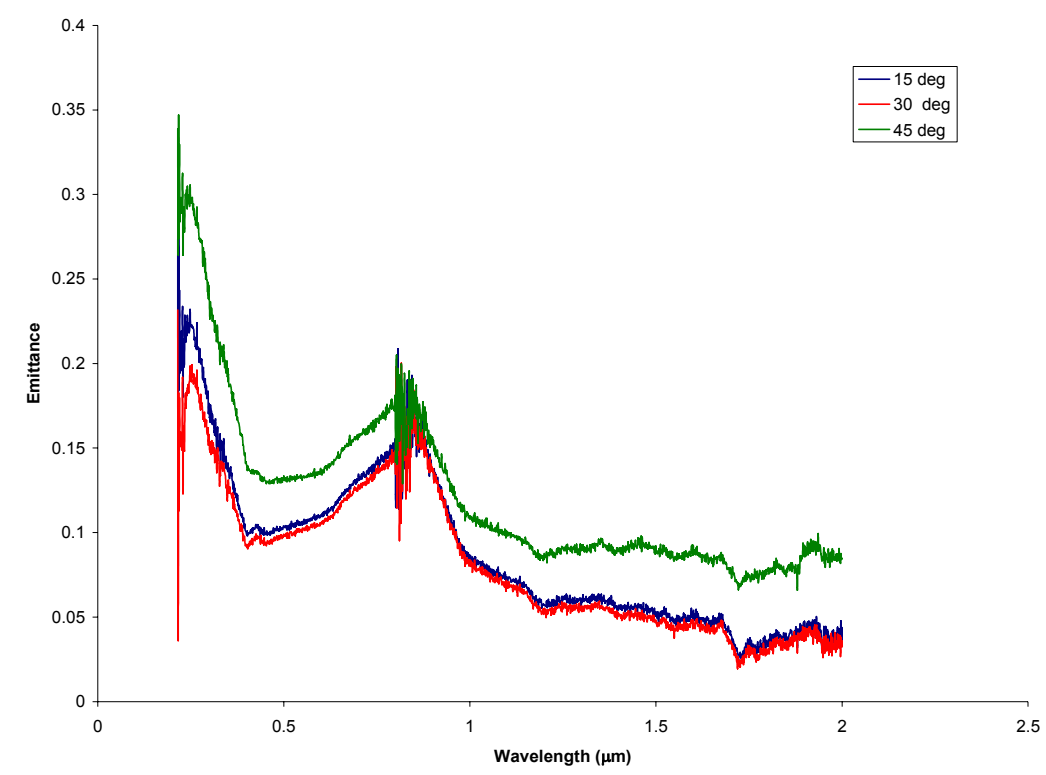

Figure 6. Emittance as a function of wavelength between 0.2 and $2 \mu \mathrm{m}$ for the aluminum face of a 1.0-mil Kapton sample with aluminum and uncoated faces. 
Figures 7 and 8 present the emittance profile in the infrared waveband for aluminum coated, and uncoated, surfaces of the Kapton sample.

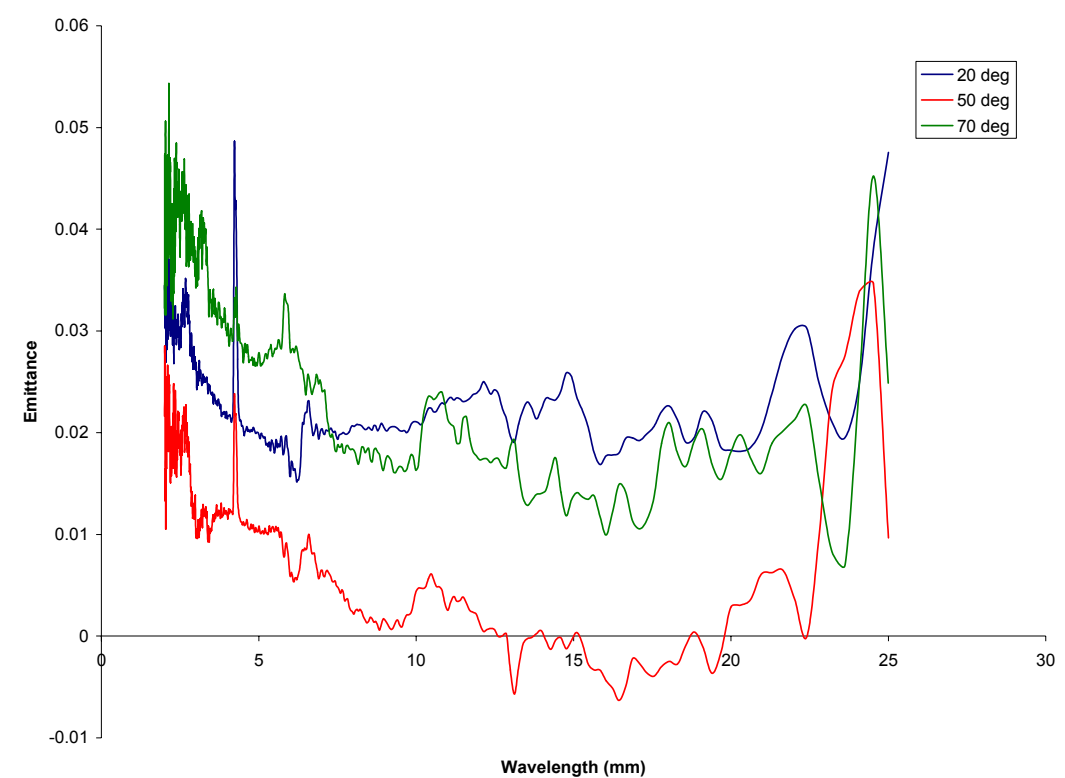

Figure 7. Emittance as a function of wavelength between 2 and $25 \mu \mathrm{m}$ for the aluminum face of a 1.0-mil Kapton sample with aluminum and uncoated faces.

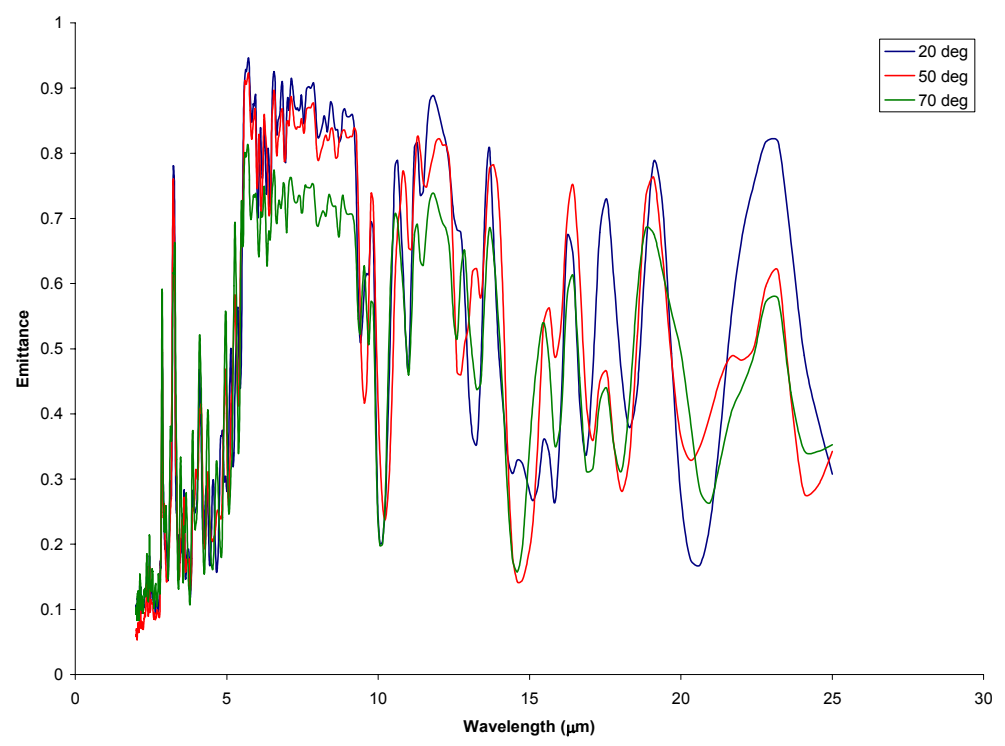

Figure 8. Emittance as a function of wavelength between 2 and $25 \mu \mathrm{m}$ for the uncoated face of a 1.0-mil Kapton sample with aluminum and uncoated faces.

Figure 9 shows the emittance profile for solar wavelengths for the Mylar sample. Figures 10 and 11 present the emittance profile in the infrared waveband for aluminum- and black chrome-coated surfaces of the Mylar sample. 


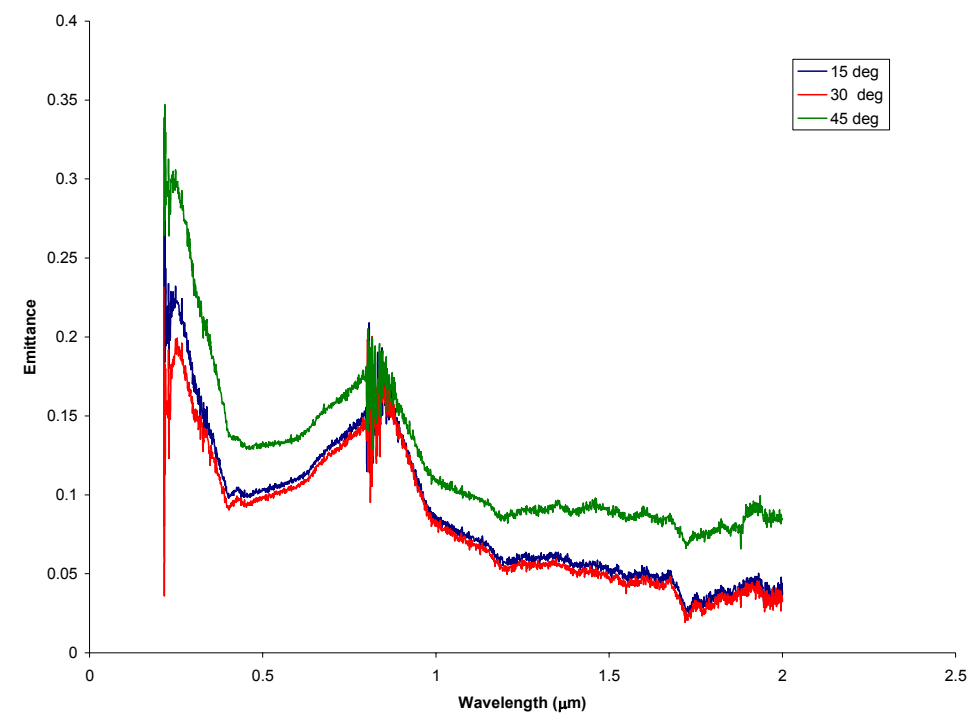

Figure 9. Emittance as a function of wavelength between 0.2 and $2 \mu \mathrm{m}$ for the aluminized face of a 0.8-mil Mylar sample with aluminum and high-emittance chrome coatings.

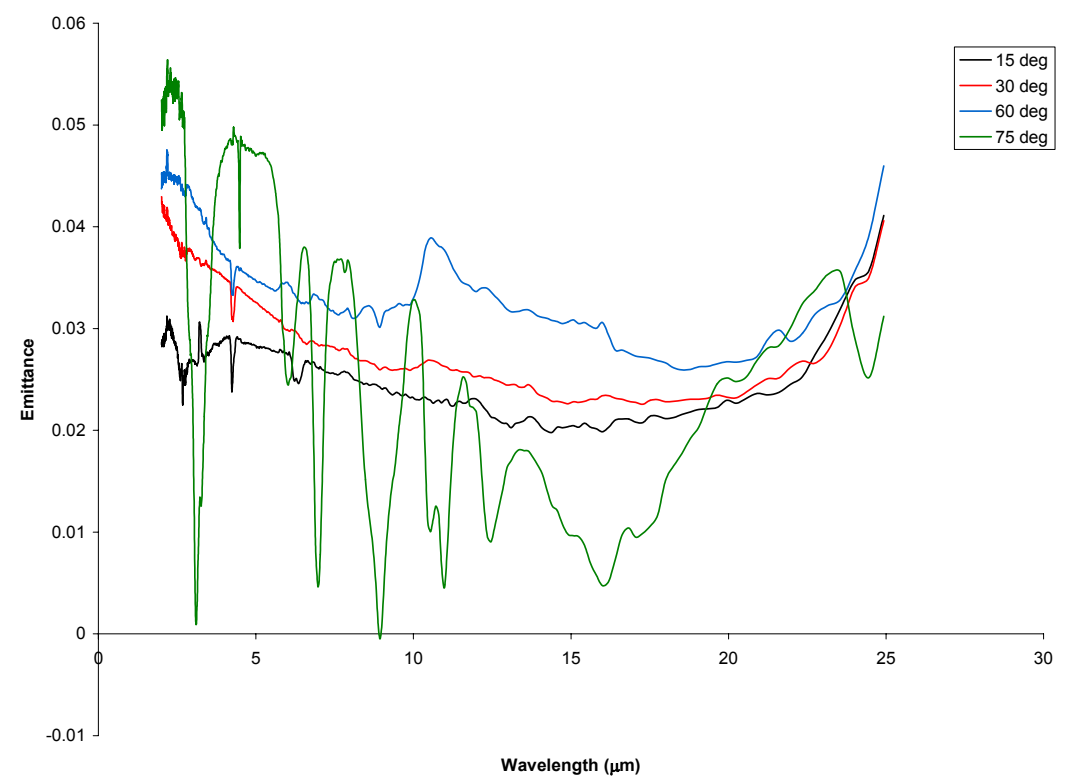

Figure 10. Emittance as a function of wavelength between 2 and $25 \mu \mathrm{m}$ for the aluminum face of a 0.8-mil Mylar sample with aluminum and high-emittance chrome coatings. 


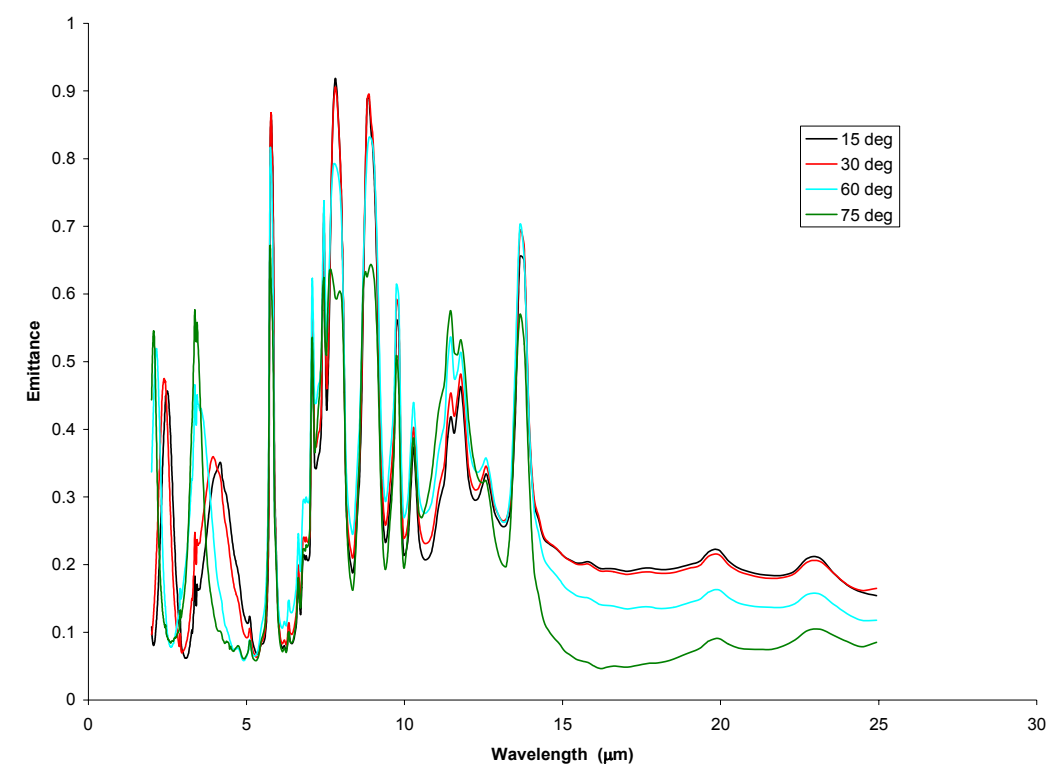

Figure 11. Emittance as a function of wavelength between 2 and $25 \mu \mathrm{m}$ for the high-emittance chrome face of a 0.8 -mil Mylar sample with aluminum and high-emittance chrome coatings.

For coated membranes of this type, the transmission of radiation through the membrane is very small, and in most cases negligible. Given the assumption of no transmission through the membrane, the law of conservation of energy suggests that at any wavelength the reflectance equals the emittance. Figures 6-11 were generated based upon this assumption. Figures 6-7 and 9-10 demonstrate that most solar photons incident upon the aluminum-coated sides of each sample are reflected at all wavelengths. The chrome-coated side of Mylar, and the uncoated side of Kapton, were they exposed to solar incidence, would absorb efficiently and therefore cause significant heating of the membrane.

Figures 10 and 11 demonstrate that the aluminum-coated side of Mylar is a very poor emitter of thermal radiation, but the chrome-coated side is a relatively efficient emitter of thermal radiation. Both coatings demonstrate significant variation with respect to wavelength. This suggests that a rigorous thermal energy balance conducted to predict steady state temperature profile of a membrane in flight should include a spectral dependence. Figures 6-8 suggest that a comparably rigorous analysis pertaining to 1.0-mil Kapton should also use spectral analysis for most reliable prediction of temperature profile.

\section{INFRARED MEASUREMENT TECHNOLOGIES}

The indigo ${ }^{\mathrm{TM}} \mathrm{Omega}^{\mathrm{TM}}$ (long-wave) miniature microbolometer-based imager with on-focal plane processing was tested to determine the feasibility of using a similar technology to acquire temperatures from a solar sail membrane. It features small size, low mass $(<0.12 \mathrm{~kg})$, and excellent image quality. It is equipped with an uncooled bolometer $\left(\mathrm{VO}_{\mathrm{X}}\right)$ with $160 \times 120$ pixels sensitive to $7.5-13.5 \mu \mathrm{m}$. It acquires images at $30 \mathrm{~Hz}$ and features a noise-equivalent delta temperature $(\mathrm{NedT})<85 \mathrm{mK}$. Its temperature detection range is -40 to $55^{\circ} \mathrm{C}$ (extended).

Infrared image data were collected from the aluminized side of a $0.5-\mathrm{m}$ (on side) Kapton sample with ambient temperature of $21.6^{\circ} \mathrm{C}$. Three imagers were placed behind a shield intended to minimize stray ambient reflections from the surroundings. An Agema Thermovision ${ }^{\circledR} 870$ (mid-wave) infrared scanning system and a CMC Electronics Cincinnati TVS-8500 (mid-wave) infrared imaging system were used for comparison during evaluation of the Omega system. These two systems are laboratory-grade instruments and the TVS-8500 represents the state of the art in Stirlingcooled, mid-wave focal plane array technology. The Thermovision system is old technology, but robust and flexible in its operation. The three imagers were stationed behind a barrier constructed to minimize stray ambient reflections. The 
indigo and Agema units were placed side by side and centered on the membrane, while the CMC unit was placed higher (height about that of top of the membrane). The membrane was heated radiatively using a circular resistive element centered behind the membrane. The images collected with the indigo and Agema units are shown in Figures 12 and 13 with no power applied and then power applied at three different levels.

Specular reflections are apparent in all the images, but are more easily distinguished in the monochrome images produced by the indigo system. At low power setting the warmed round region begins to become discernible in the center of the membrane, and in the images corresponding to medium and high power setting (Figure 13) the effects of natural convection become apparent. The Agema system calculates target temperature based upon a proprietary algorithm that considers three inputs from the user: $T_{\text {ambient }}$ (temperature of the surrounding atmosphere), $T_{\text {reflected }}$ (a nominal average blackbody temperature of the objects surrounding the target), and $\varepsilon$ (an average emissivity value pertaining to the target). The annotations in blue shown in Figures 12 and 13 demonstrate the sensitivity of a "true" temperature calculation to the average emissivity value assumed for calculation.

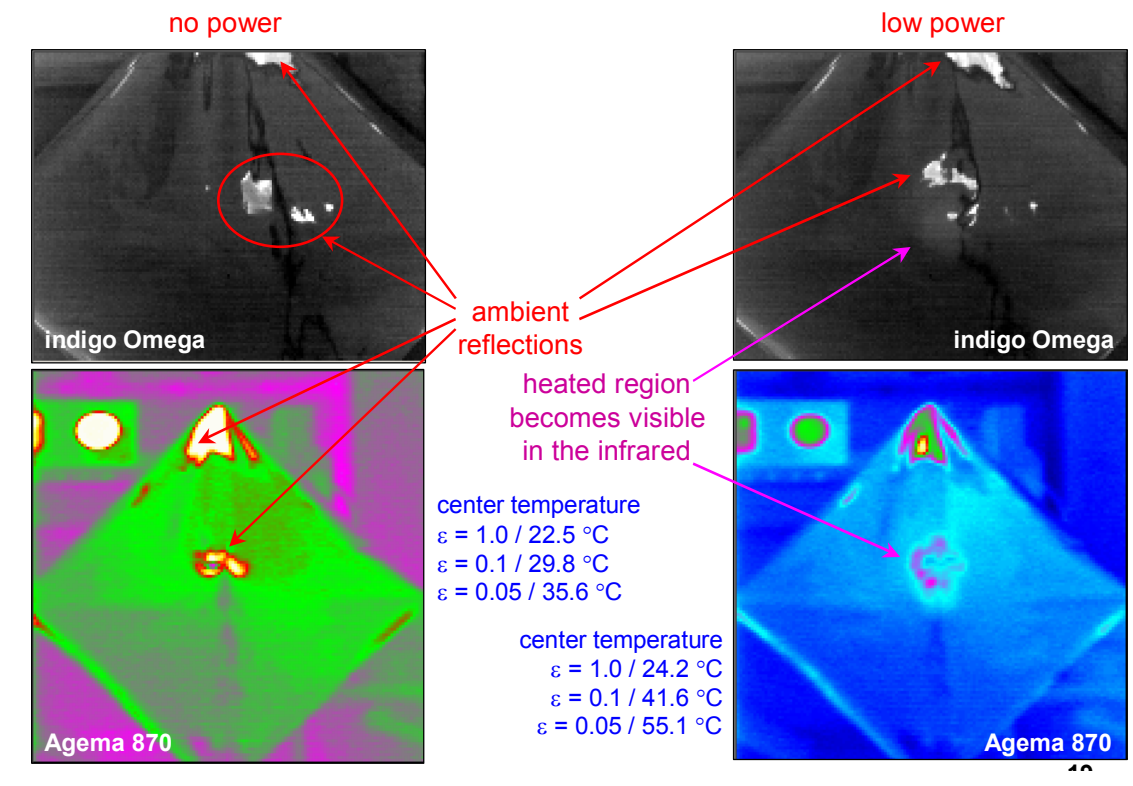

Figure 12. Infrared images of 0.5-m (on side), 1-mil aluminized Kapton sample at ambient temperature (left) and with low power applied at center (right).

Although these results demonstrate that radiometric measurement of the aluminized side of a coated membrane is possible, the data were acquired under carefully controlled conditions. The primary advantage to back side thermography is that a significantly stronger infrared signal is available given the overall higher emittance profile on the uncoated or black chrome-coated side, either of which would be on the back, or shadow, side of a sailcraft. Furthermore, the effects of reflections from other objects, especially for a flight that is relatively close to earth, are diminished when imaging from the side that is less reflective. A preliminary study with a sample membrane illuminated by a solar source in the laboratory confirmed that uncoated- and black chrome-side thermography is feasible using an uncooled bolometer under typical flight conditions. 

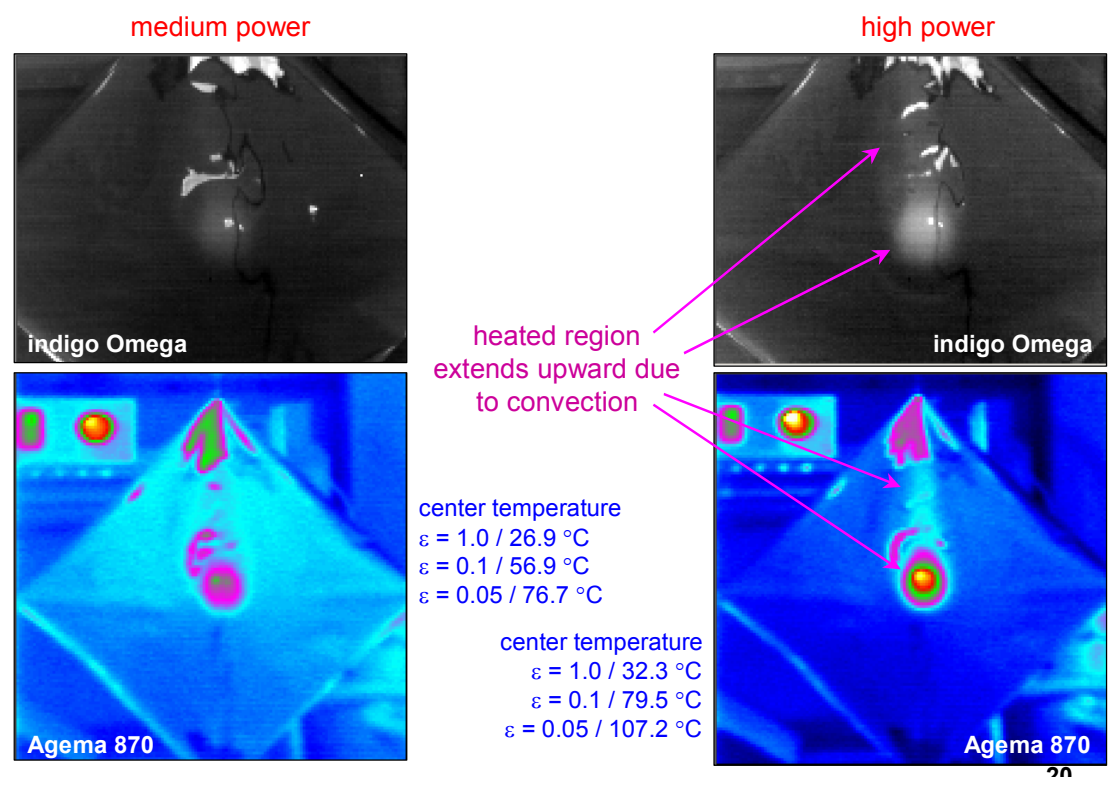

Figure 13. Infrared images of 0.5-m (on side), 1-mil aluminized Kapton sample with medium power applied at center (left) and with high power applied at center (right).

\section{CONCLUSIONS}

The deployment and flight of a sailcraft will subject the membrane structure, the principal structure responsible for propulsion, to significant thermal and mechanical strains that can, in turn, impact the dynamic behavior and controllability of the vehicle. An Optical Diagnostics System will provide during test flights the means to image in the infrared and to measure the temperature profile across the membrane. The optical properties of either the coated or the uncoated side of a polymer substrate can vary significantly with wavelength. Therefore, a thermal model that performs an energy balance to predict the in-space temperature profile, for the purposes of estimatiing thermal strains and defining the operating parameters of a measurement system, should incorporate a wavelength-dependent analysis. Such a thermal model has been developed and tested, and several candidate materials for solar sails have been tested to characterize their optical properties. An uncooled microbolometer-based imager has been demonstrated to acquire temperatures from sample membrane materials with performance that is comparable to that of a traditional cooled imager.

\section{ACKNOWLEDGEMENTS}

The work described in this paper was funded in part by the In-Space Propulsion Technology Program, which is managed by NASA's Science Mission Directorate in Washington, D.C., and implemented by the In-Space Propulsion Technology Office at Marshall Space Flight Center in Huntsville, Ala. The program objective is to develop in-space propulsion technologies that can enable or benefit near and mid-term NASA space science missions by significantly reducing cost, mass, or travel times. This effort was conducted in the Infrared Development and Thermal Structures Laboratory at James Madison University. The authors thank Ms. Stefanie Bourne and Mr. Joshua Krause for their considerable contributions. A special thanks as well to our friends at Surface Optics Corporation for conducting solar reflectance measurements on our behalf. 


\section{REFERENCES}

1. Kreith, F., Radiation Heat Transfer for Spacecraft and Solar Power Plant Design, International Textbook, Scranton, PA, 1962, pp. 95-96.

2. Wertz, J.R., and Larson, W.J., Space Mission Analysis and Design, Kluwer, Dordrecht, The Netherlands, 1991, pp. 370-386.

3. Fortescue, P.W., and Stark, J.P.W., Spacecraft Systems Engineering, Wiley, New York, 1991, pp. 275-302.

4. Griffen, M.D., and French, J.R., Space Vehicle Design, Education Series, AIAA, Washington, DC, 1991, pp. 371394.

5. Cengel Y.A. and Turner, R.H., Fundamentals of Thermal Fluid Sciences, $2^{\text {nd }}$ Edition, McGraw Hill, Boston, 2005, pp. 974-975.

6. Planck, M., The Theory of Heat Radiation, Dover Publications, New York, 1959.

7. Siegel, R., and Howell, J.R., Thermal Radiation Heat Transfer, $2^{\text {nd }}$ Edition, McGraw Hill, New York, 1981.

8. Jenkins, C.H.M. Editor, Gossamer Spacecraft: Membrane and Inflatable Structures Technology for Space Applications, Progress in Astronautics and Aeronautics Series, AIAA, Washington, DC, 2001.

9. McInnes, C.R., Solar Sailing Technology, Dynamics and Mission Applications, Praxis Publishing, Chichester, UK, 1999.

10. Fay, C.C., Stoakley, D.M., St. Clair, A.K. (1998). Molecularly oriented polymeric thin films for space applications. NASA Langley Research Center, Materials Division, Hampton, VA. 